\title{
ROSAT X-RAY OBSERVATIONS OF THE RADIO GALAXY 4C46.09
}

\author{
D.A. LEAHY \\ University of Calgary \\ Calgary, Alberta, Canada T2N $1 N_{4}$
}

\section{Introduction}

4C46.09 is the radio source that shows up as a point-like $\mathrm{x}$-ray source inside the supernova remnant HB9 (Leahy, 1987). Leahy, 1987 found a 0.2$4 \mathrm{keV}$ Einstein IPC flux of approximately $1.5 \times 10^{-12} \mathrm{erg} \mathrm{cm} \mathrm{cm}^{-2} \mathrm{~s}^{-1}$ and a significantly higher hardness ratio than the rest of HB9. Too few counts were available for any spectral analysis. Seward et al, 1991, found 4C46.09 to be a large radio galaxy at redshift 0.195 and distance $1280 \mathrm{Mpc}\left(H_{o}=\right.$ $\left.50 \mathrm{~km} \mathrm{~s}^{-1} \mathrm{Mpc}^{-1}\right) .4 \mathrm{C} 46.09$ is of further interest due to the observation of a high energy component in the spectrum of HB9 observed by GINGA (Yamauchi and Koyama, 1993). Whether this was due to HB9 or to 4C46.09 could not be determined.

4C46.09 is the only 4C source inside HB9. and is clearly seen in the radio maps of HB9 of Leahy and Roger, 1991, i.e. at $408 \mathrm{MHz}$ (their Fig. 1) and $1420 \mathrm{MHz}$ (their Fig. 2). The radio flux densities at 408 and 1420 $\mathrm{MHz}$ (Leahy and Roger 1995) are $1480 \mathrm{mJy}$ and $248 \mathrm{mJy}$, respectively. Its position is (1950) R.A. $4^{h} 54^{m} 45.61^{s}$, decl. $46^{\circ} 19^{\prime} 58.0^{\prime \prime}$ (Seward et al, 1991).

The current work has purpose to clarify whether the hard tail observed by GINGA is due to $4 \mathrm{C} 46.09$ or to HB9, and also to learn more about 4C46.09. The ROSAT All-Sky-Survey data of the HB9 region is re-examined here with an analysis of the soft $\mathrm{x}$-ray spectrum of $4 \mathrm{C} 46.09$.

\section{Observations and Data Analysis}

The data was obtained from the ROSAT All Sky Survey data base at MPE, Garching and analyzed at MPE using the EXSAS software. The ROSAT map of the region is dominated by HB9 (Leahy and Aschenbach, 1995), and 4C46.09 is not easily seen within HB9. However there is a bright 
region associated with the known coordinates of $4 \mathrm{C} 46.09$. This bright region coincides with northwest "arm" of the bright cross-shaped region in the western half of HB9. 4C46.09 shows up as a high hardness ratio region inside the western boundary of HB9 in the the hardness ratio $(0.4-0.9 \mathrm{keV} /$ 0.9-2.4 keV) map of HB9.

Spectral fitting to the background subtracted source spectrum was carried out for a Raymond-Smith model. The best fit values are $\mathrm{kT}=1.7$ $\mathrm{keV}$ and $N_{H}=6 \times 10^{20} \mathrm{~cm}^{-2}$. The emission measure for the best fit is $3 \times 10^{42} \mathrm{~cm}^{-5} \times D^{2}$, with $\mathrm{D}$ in $\mathrm{kpc}$. The $0.1-2.4 \mathrm{keV}$ flux from $4 \mathrm{C} 46.09$ is $1 \times 10^{-10} \mathrm{erg} \mathrm{cm}^{-2} \mathrm{~s}^{-1}$.

\section{Results and Discussion}

The hard x-ray emission from 4C46.09 and HB9 has been previously discussed by Yamauchi and Koyama (1993) in non-imaging observations of HB9 with the LAC detectors on GINGA. The GINGA $2-10 \mathrm{keV}$ flux of $6 \times 10^{-12} \mathrm{ergcm}^{-2} \mathrm{~s}^{-1}$ for the $4 \mathrm{C} 46.09$ plus HB9 (the LAC field of view is just larger than HB9, see their Figure 1). Based on the spectral fits here, this flux is consistent with an origin entirely from $4 \mathrm{C} 46.09$.

The 0.1-2.4 keV x-ray luminosity of $4 \mathrm{C} 46.09$ for a distance of 1280 $\mathrm{Mpc}$ is $6 \times 10^{46} \mathrm{erg} \mathrm{s}^{-1}$, which makes it rather bright for an AGN. The optical luminosity of $4 \mathrm{C} 46.09$ (Seward et al 1991) is $5 \times 10^{11} L_{\text {sun }}$ and the optical spectrum is dominated by starlight. Since an AGN nucleus does not dominate the optical light, the x-ray luminosity is not likely to be from a bright AGN but rather is probably due to diffuse emission from the cluster. The $\mathrm{x}$-ray emission measure gives $n_{e} n_{H} V / D^{2}$. For a cluster of $1 \mathrm{Mpc}$ radius, one finds $n_{e} n_{H}=3 \times 10^{-7} \mathrm{~cm}^{-6}$, which is characteristic of diffuse cluster $\mathrm{x}$-ray emission.

In, summary, it is found that the x-ray emission from $4 \mathrm{C} 46.09$ may be due to diffuse emission from the cluster, and that it can account for the hard $x$-ray emission observed from the HB9/4C46.09 region by GINGA.

References

Leahy, D. 1987, ApJ, 322917

Leahy, D., and Roger, R. 1991, AJ, 102, 2047

Leahy, D., and Roger, R. 1996, A\&A Supp., 115, 345

Leahy, D. and Aschenbach, B. 1995, A\&A, 293, 853

Seward, F. et al 1991, AJ, 102, 2047

Yamauchi, S. and Koyama, K. 1993, PASJ 45, 545 